\title{
Research on Innovative Strategies of Accurate Development Model of Regional Agricultural Economy under Big Data Coordination
}

\author{
Bin Li, Ni Yang \\ Sichuan Aerospace Vocational College Chengdu, Sichuan 610100
}

\begin{abstract}
This article will analyze the correlation between big data and regional agricultural economic development on the basis of fully understanding the connotation and characteristics of big data technology. Then, we should use its concept and related technologies and operating models to realize the precise operation guidance of multiple links such as agricultural product planting, selection, planting, farm management, harvesting and storage, and marketing. Through the integration of big data and local advantageous agricultural resources, it is possible to build a fusion and innovation mechanism based on big data, and build an open, intelligent, and intensive precision agriculture development format. This can accelerate the realization of agricultural informatization and modernization, and promote the innovative development of regional agricultural economy.
\end{abstract}

\section{Introduction}

With the development of computer technology, a variety of new information technologies have emerged and been widely used in various industries such as big data, Internet of Things, cloud computing and so on. The application of these new technologies to agricultural production management can not only realize the collection, integration and analysis of massive agricultural production data, but also facilitate the construction of an agricultural production model suitable for local development that can give full play to local advantages. It can provide guidance for the precision development of regional agriculture on the basis of increasing production and reducing expenditures and improving farmers' benefits. It can accelerate the realization of the precise development goal of regional agricultural economy through the rational allocation of various production factors. The integration of big data concepts, technologies and operating models with regional agriculture can achieve innovation at the operational level by constructing big data-based innovation mechanisms for big data and regional agricultural economic development, following the principles of equality, openness, and win-win cooperation. Moreover, computer technology can build a modern agricultural production information collection and management mechanism based on regional differences and diversity. At the same time, it can simultaneously optimize related work such as agricultural business entities, agricultural product production and processing, agricultural technology promotion and farmer training, and give full play to the advantages of big data technology in driving local agricultural economic development from the actual development of local agricultural economy. This will not only achieve the goal of increasing production and income, but also activate the vitality of the market.

\section{Big Data Overall Planning Method to Support the Precise Development of Regional Agricultural Economy}

\subsection{Precisely Guide the Market Forecast of Regional Agricultural Planting}

affected by weather, regional natural disasters, and overall economic development. Therefore, we should take the overall planning mechanism of collecting, analyzing and predicting big data as the focus of development, and create good conditions for the precise operation and development of the regional agricultural economy. If we want to build a model of precise regional agricultural economic development based on big data, first of all, we need to use big data to complete market forecasts. That is, it can realize a comprehensive forecast of the regional agricultural economic development according to the laws of the market, and adopt big data on agricultural production from relevant government departments and various research institutions. This can accurately guide the selection, planting and marketing of various crops, thereby ensuring the stable and healthy development of the agricultural economy in the region. Specifically, for one thing, we need to accurately guide

\footnotetext{
*Corresponding author: 2003408425@dlvtc.edu.cn
} 
the supporting planting of crops to avoid that the crop types in a certain area are too single, which is not conducive to later development and affects the agricultural economic development of the entire region. Under the guidance of big data on crop characteristics, we can realize the function of mutual promotion between different crops on the basis of analyzing the local natural environment characteristics and the production characteristics of various crops, and the requirements for production. We can use the natural pollination between crops, and one crop can treat other one or more diseases and insect pests to accurately guide the joint development of regional agricultural advantages. In addition, we should accurately guide the planting volume of crops and choose the planting scale reasonably according to the law of market development. In this way, economic losses caused by blindly following the trend of investment in planting can be avoided, and reasonable investment and planting can be guided to respect market laws.

\subsection{The Agricultural Index that Accurately Leads the Production of Advantageous Agricultural Products}

Regional agricultural economic development will be affected by various domestic factors and international events, thus forming a comprehensive international and domestic dynamic index. For example, the meteorological data of the National Meteorological Department and the market business forecast index of the Ministry of Commerce can provide precise guidance on the development of agricultural products from the international and domestic macro perspectives. For one thing, we apply big data to accurately guide the information flow and capital flow required for regional agricultural production, according to the overall cultivated land area and the planting area of various crops, crop planting types and planting techniques, as well as the market of crops and raw products and processed products. And other data to accurately judge agricultural production and agricultural product information. This will bring more precise capital flow to the development of regional agricultural economy, and achieve the goal of multi-party and multi-win based on support and service production. For another, the use of big data can accurately determine the proportion of original products, processed products, and deeply processed products of regional agricultural products, open up a diversified market, and successfully guide the diversified development of agricultural products.

\subsection{Accurately Integrate Technical Parameters of Agricultural Resources}

The regional agricultural economy must be supported by technology in order to obtain fast and good development. In the context of big data, the upgrading and optimization of the agricultural industry structure must be based on the realization of resource optimization and integration. First, we should use big data to realize the integration of land resources. According to the requirements of rural construction and comprehensive land management, we can integrate the land of Hollow Village, abandoned factories and farm houses, and treat ponds and river courses. Moreover, we can also build new large farms and rural cooperatives based on the protection of arable land and combine the actual carrying capacity of resources to build a more comprehensive agricultural production model to maximize the use of land resources and maximize production capacity. Otherwise, we should use big data to realize the integration of agricultural production technology, and choose the most optimized production technology on the basis of making full use of land resources. These technologies include mechanization and information farming technologies, sewage treatment technologies under three-dimensional farms, agricultural products storage and primary processing technologies, and ecological landscape technologies. They can fully achieve the goals of efficient agricultural economic development and green environmental protection.

\section{Relevance Analysis and Relevance Test of Big Data and Precise Development of Regional Agricultural Economy}

\subsection{Analysis of the Correlation Between Big Data and the Precise Development of Regional Agricultural Economy}

The strength of the correlation between big data and the precise development of the regional agricultural economy directly determines whether big data can be used to promote the precise development of the agricultural economy. If the correlation is strong, it can provide a theoretical basis for using big data to promote the precise development and innovation of regional agricultural economy. When conducting big data analysis, we found that big data has the characteristics of larger data scale, faster circulation speed, more data types and lower value density. This is not only its characteristic but also the basis for measuring whether a certain region's agricultural development has the characteristics of big data. First of all, the scale of product data in regional agricultural economic development is relatively large, and the types and characteristics of agricultural products in different regions are different, and the industrial structure layout is complicated. Second, the data sources are diverse. Among them, it includes structured data and unstructured data, and the data comes from multiple provinces, municipalities and districts across the country, covering data from various departments. Thirdly, big data flows very fast. Not only is the speed of data generation fast, but the mobility between regions is also fast. The collection and processing of these data in the agricultural economy is due to different regional standards. In addition, it is limited by manpower and data scale, so it is easy to have a large gap between the final data and the real data, which increases the workload of supplements 
and reviews. Furthermore, the value density of data is low. There is independence between the decentralized storage of China's agricultural economic data and regions, which restricts the generation and use of big data. The decentralized data processing method is not only costly, but also easy to be distorted. If the next step is not processed, it will lose its use value.

\subsection{Relevance Test of Big Data and Precise Development of Regional Agricultural Economy}

\subsubsection{Data Collection}

At the present stage, data collection on agricultural economic development in most regions mainly uses questionnaire surveys and on-site visits. Most of these data are structured data and are basically manually entered. Few of them are directly and automatically collected using Internet terminals, and they specify the time limit for completing tasks at various stages. Because of the limitation of hardware equipment, the degree of automation of data collection is very low, and the workload is large, and the timeliness of data collection is not high. In this way, data redundancy, missing data, and poor reliability are prone to problems.

\subsubsection{Data Storage}

The precise development of the agricultural economy requires the collection of data from various departments. Then, we can build a big data platform to mathematically model industry databases, industry databases, and social databases. In this way, the processing, sorting, tracking and display of data at various stages can be realized, and performance evaluation can be completed at the same time. However, because the data scale is less flexible and has poor scalability, it cannot form an effective connection with big data technology. It has certain deficiencies in the scale and diversity of data.

\subsubsection{Data Analysis}

Regional agricultural economic data includes attribute data and non-attribute data. We can use geographic information systems to realize real-time monitoring of agricultural economic development in the region. In order to test the performance of the precise development of the agricultural economy, we have established corresponding indicators and quantified them, so as to monitor the entire development process, so that we can comprehensively use a variety of big data technologies to achieve real-time data, supervision and performance evaluation of various types of data. It also can provide a basis for the integration between big data and the precise development of the regional economy. But as far as the whole country is concerned, China is currently not very mature in the application of neural networks, random forest and other algorithm technologies. We also need to strengthen the research and application of technology in order to further realize the in-depth mining of the big data of agricultural economic development and unearth the complex variable relationships between big data. Afterwards, we should build an analytical model to discover the internal connections between variables and predict the performance of development projects.

\section{Performance Evaluation of Regional Agricultural Economic Precision Development Model Under Big Data Coordination}

\subsection{Upgrade and Optimization of Technical Equipment to Achieve the Goal of Increasing Income}

If we want to promote the sustainable development of the regional agricultural economy, we must make good use of big data technology to guide the relevant technology of regional agricultural economic upgrading and optimization, and then achieve the goals of efficient production, environmental protection, and resource conservation. Among them, the environmental protection and ecological technologies under the overall planning of big data include technologies such as pest control, production and utilization of miscellaneous fertilizers, and straw utilization. Resource-saving technologies include drip irrigation technology, three-dimensional planting technology, and mechanized harvesting technology. It can not only increase the output, but also reduce the consumption of resources, achieving the goal of higher quality, more output and economic profit of agricultural products. The performance during performance evaluation is as follows. Firstly, its input becomes smaller and its output becomes larger. Including the reduction of human, material and financial resources, the use of fertilizers and pesticides has decreased. This not only saves costs but also benefits environmental protection. However, the actual output of agricultural products is increasing, the quality is improved, the net profit of the farm is increased, and the income of the farmers is also increased. Secondly, the varieties of crops have been upgraded and optimized, and the ecological environment has been improved. The regional agricultural economy can be upgraded to focus on the varieties of agricultural products and drive the improvement of rural ecology, thereby realizing a virtuous circle of ecology and production.

\subsection{Upgrading and Optimizing the Industrial Structure, Improving the Ability of Risk Prevention and Control}

For the precise development of regional agricultural economy under the coordination of big data, performance evaluation should not only evaluate the actual input-output ratio, the quality of agricultural products and market profits, but also the entire agricultural industry and the ability to control various risks. We can design a regional agricultural structure upgrade plan based on the regional agricultural economic development 
plan of a specific administrative division government on the basis of considering the economic level of each region, the total number of rural and agricultural populations, the importance of farmland protection, and the cultural level. Firstly, we should upgrade the structure of regional crop varieties, the structure of production and processing. For example, upgrading early and late maturing crop varieties and their proportions, upgrading the original products of agricultural products and the structure of processed products of different levels. In this way, it can reduce the loss caused by the overproduction of the original agricultural product structure caused by the single structure, and the reduction of production or even the loss of production during natural disasters. What's more, we should use big data to accurately guide the scientific prevention of regional agricultural economy. Especially for the prevention of natural disasters and crop diseases and insect pests, we can use weather forecast data to avoid fruit blooming cold currents in extreme weather, and use mountain safety monitoring data to avoid possible economic losses caused by natural disasters such as mudslides and landslides. Moreover, the use of pest forecast data can effectively prevent pests and diseases and provide guarantee for the normal production of crops.

\section{The Construction of A Big Data Platform under the Innovation of the Integration Mechanism of Big Data and the Precise Development of Regional Agricultural Economy}

\subsection{Data Collection Automation}

The function of the data collection automation module is to realize the automatic collection of large-scale data. Its characteristic is to shorten the time of data collection and improve the speed of data integration. It can realize the integration of basic information and develop application software for the precise development of agricultural economy. Use mobile devices to integrate data collection into applications anytime, anywhere. Furthermore, building a regional e-commerce platform and building a supply and marketing channel for agricultural products can not only increase the income of agricultural products, but also grasp the income and expenditure data of farmers in time.

\subsection{Data Sharing}

Building a big data cloud platform can realize the integration of agricultural economic development data in various regions of the country, so that all kinds of data from different departments and different regions can be interconnected and shared in real time. So as to be able to develop the regional agricultural economy and performance appraisal more comprehensively and accurately.

\subsection{Market Risk Warning}

The big data platform realizes real-time sharing of information across regions, and it can transform the results of market supply and demand data processing to reliable market risk diagnosis results through risk analysis techniques and theories. Thus, risks can be detected in time and an early warning can be issued. We can use data mining technology to identify the key indicators in the regional agricultural economic development big data, and then use principal component analysis and correlation analysis to dynamically monitor and evaluate the market risks of agricultural products, labor, and other production factors. In this way, we can timely provide risk information and risk response measures formed by the expert group discussion to farmers, which can minimize economic losses.

\section{Innovative Model of Precise Development of Regional Agricultural Economy Under the Coordination of Big Data}

\subsection{Relying on Local Resources to Adjust Crop Planting to Accelerate the Realization of the Goal of Precise and Characteristic Development of Agricultural Products}

The scientific and reasonable use of big data platforms can not only promote the precise development of regional agricultural economy, but also highlight the characteristics of regional agricultural economic development and optimize agricultural operation and development models. Under the big data platform, we should build an innovation mechanism for the integration of big data and regional agricultural economy. Relying on local resources, we can first realize the rational adjustment of crop planting structure and realize the precise and characteristic development of products. That is, the use of national soil testing soil composition big data and regional meteorological big data, according to the development status of various crops at home and abroad, product data and market data, etc., provide a basis for the selection of farms and varieties, so as to achieve unique and precise development. The staff of the government's agricultural department, science and technology department and other related departments are responsible for technology provision and supervision and control. For example, the use of science and technology to achieve the detection of regional plot soil, the collection and analysis of meteorological data. Then, we can choose appropriate crop varieties according to local conditions, and adopt targeted planting production management plans to provide technical support for the development of regional crop characteristics. 


\subsection{Create An Interactive Crop Chain to Promote the Precise and Mutually-assisted Development of Multiple Crops}

The use of big data can realize the collection and integrated analysis of regional agricultural soil, climate, topography and other elements, so as to accurately determine which types of crops are suitable for planting in the region. With the support of modern science and technology, we can establish a chain of advantageous crops that can help each other develop, so that crops can promote each other's growth, thereby breaking the shackles of enthusiasm planting of crops and avoiding the problem of land resources being idle and wasted. At the present stage, the ideal crop chain models include paddy and dry rotation and three-dimensional fishery. In this way, the unified goal of improving resource utilization and agricultural output efficiency and the goal of increasing farmers' income and improving social benefits are achieved.

\subsection{Improve the Industrial Structure Layout and Promote the Precise and Coordinated Development of Multiple Industries}

Under the coordination of big data, the big data platform can be used to realize the integration and integration of modern agriculture, processing industry, modern agricultural product marketing service industry, rural tourism industry and modern rural pension industry. At the same time, the use of big data platforms can make scientific allocations for the healthy, stable and sustainable development of regional industries. From a macro perspective, the use of big data on agricultural economic development has broken the path selection of traditional agricultural development. Through the establishment of a more complete farmer skills training system, it attaches importance to the unification of the leading role of new business entities in the behavior of small farmers. In the process of using big data to promote the development of local agricultural economy, we can take the development of local advantageous agricultural products and their marketing and processing as the leading elements, and promote the coordinated development of related industries as the core. This will enable other related industries to provide technical, resource, and human support for the development of agriculture. Meanwhile, the development of agriculture can also provide a source of power and raw materials for the development of other industries, achieving the goal of mutual support and coordinated development.

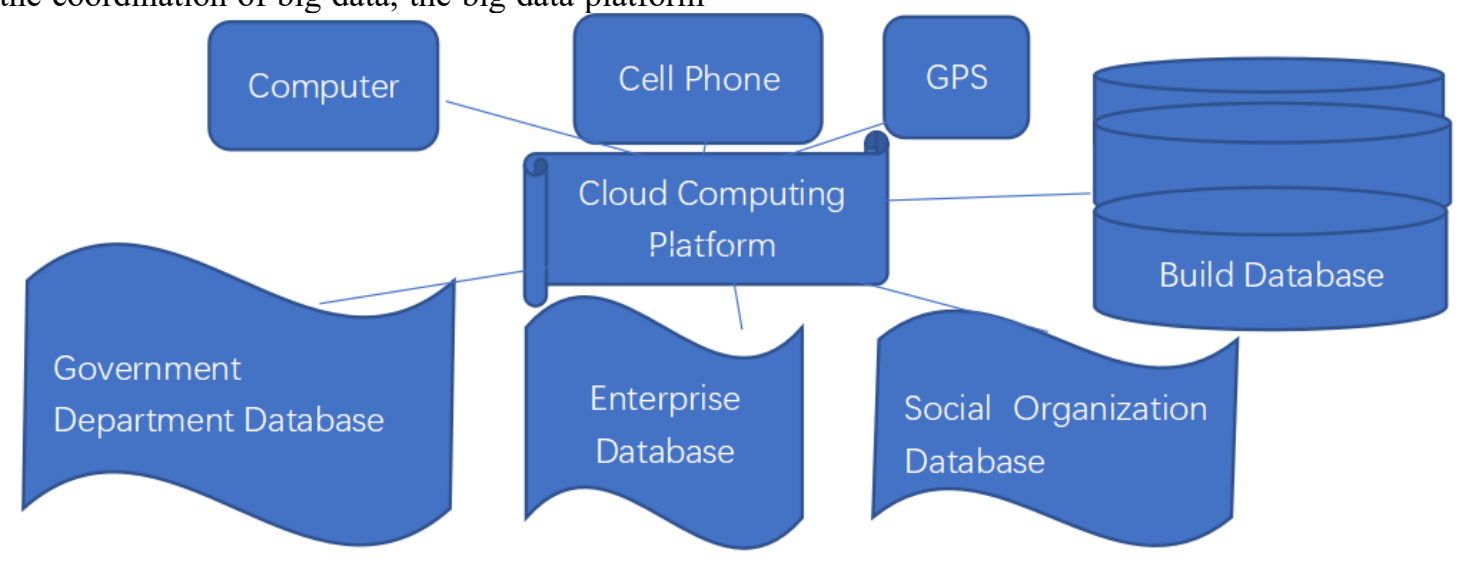

Figure1: Architecture of A Management Platform for the Precise Development of Regional Agricultural Economy Based on Big Data

\section{Conclusion}

In summary, in order to achieve the goal of precise development of regional agricultural economy, we can build a systematic and long-term comprehensive project platform under the coordination of big data. In addition, we can also build an innovative mechanism that integrates big data and agricultural economic development, leverage the advantages of all parties in society, and establish a development trend of information sharing, multi-industry joint development, and win-win cooperation. In this way, not only can the market's role in the basic allocation of resources be brought into play, but also through the joint efforts of various departments of local government. We can use big data to guide and monitor regional agricultural economic development, and actively collect and research relevant data, so as to achieve scientific, reasonable, sustainable and stable precision for achieving the goal of more efficient, stable and sustainable development of the regional agricultural economy guide. This is conducive to realizing the goal of modernized new countryside construction with industrial optimization and upgrading, multi-industry joint development, ecological livability and improvement of farmers' living standards.

\section{References}

1. Chen Ren'an. Evaluation of the coordination between agricultural resources, environment and regional economy in Chongqing $[\mathrm{J}]$. China Agricultural Resources and Regional Planning, 2018 (4): 193-197.

2. Liu Ziyu, Xiao Jing. Construction of rural e-commerce model in Jilin Province based on cloud 
computing $[\mathrm{J}]$. Journal of Agricultural Sciences, 2018 (7): 83-88.

3. Ding Kaixin, Han Xili. Rural landscape planning in hilly areas based on the theory of complex ecosystems[J]. Journal of Ecological Environment, 2018(7): 151-158.

4. Yu Zhigang, Zhang Liang. Farmers' planting structure adjustment willingness and behavior differences: based on a survey of 341 corn farmers in Heilongjiang Province [J]. Journal of Nanjing Agricultural University, 2018 (4): 142-150,165.

5. Huang Ruhua, Wang Chunying. Investigation and analysis of the status quo of my country's government data open platform[J]. Information Theory and Practice, 2016 (7): 50-55. 